\title{
Diseño y análisis psicométrico de un instrumento para detectar presencia de ciberbullying en un contexto escolar
}

\author{
Abel Baquero Correa* y Bertha Lucía Avendaño Prieto \\ Universidad Católica de Colombia
}

(Recibido 10 Abril, 2015; Aceptado 22 Junio, 2015)

\begin{abstract}
RESUMEN: El objetivo de la presente investigación fue diseñar un instrumento para detectar Ciberbullying en un contexto escolar y analizar sus propiedades psicométricas. Los participantes fueron 299 adolescentes (54,2\% mujeres y 45,8\% hombres) con edad promedio de 15 años, pertenecientes al estrato bajo $(22,1 \%)$ y estrato medio (78\%). Se llevó a cabo un estudio cuantitativo con un diseño no experimental de tipo instrumental y corte transversal. Bajo la teoría clásica de los test, se obtuvo una adecuada consistencia interna (Alfa de Cronbach $\alpha=.864$ ) así como validez convergente con las otras medidas. Se llevó a cabo el análisis factorial exploratorio en el SPSS versión 21, el cual arrojó tres factores. Desde la teoría de respuesta al ítem, se encontró que el INFIT de los ítems osciló entre .73 y 1.23 y el OUTFIT entre 0.74 y 1.24 . Con base en los resultados favorables del análisis psicométrico, se concluye que el instrumento puede ser usado para la detección del ciberbullying en un contexto escolar.
\end{abstract}

Palabras clave: acoso escolar, bullying, validez, confiabilidad.

\section{Design and psychometric analysis of an instrument to detect the presence of cyberbullying in a school context}

\begin{abstract}
The objective of this research was to design an instrument to detect cyberbullying in a school context and analyze its psychometric properties. Participants were 299 adolescents (54.2\% female and $45.8 \%$ male) with a mean age of 15 years, belonging to the lower stratum (22.1\%) and middle layer (78\%). A quantitative study with a non-experimental design instrumental and the cross section was performed. Under the classical test theory, adequate internal consistency (Cronbach's alpha $\alpha=.864$ ) and convergent validity with other measures was obtained. Confirmatory factor analysis in SPSS version 21, which resulted in three factors was performed. From item response theory, INFIT found that the items ranged from 0.73 and 1.23 and between 0.74 and 1.24 OUTFIT. Based on the favorable results of psychometric analysis, we conclude that the instrument can be used for detection of cyberbullying in a school context.
\end{abstract}

Keywords: bullying, validity, reliability.

\section{ACOSO ESCOLAR, BULLYING Y CIBERBULLYING}

El acoso escolar también conocido como hostigamiento escolar, violencia escolar o su equivalente en inglés bullying, es un concepto introducido por Olweus 
(1998) para referirse a cualquier forma de maltrato psicológico, verbal o físico producido entre escolares de forma reiterada a lo largo de un tiempo determinado, que consiste específicamente en una especie de tortura metódica y sistemática en la que el agresor somete a la víctima a menudo con el silencio, la indiferencia o la complicidad de otros compañeros (San Martín, 2007).

Olweus (1998), desarrolló el primer criterio para identificar la forma específica de acoso escolar, cuando se descubrió que el fenómeno se asocia con alta tasa de intentos suicidas entre los adolescentes y definió una situación de acoso como aquella en la cual "un alumno es agredido o se convierte en victima si está expuesto, de forma repetida y durante un tiempo a acciones negativas que lleva a cabo otro alumno o varios de ellos". Para este autor en el acoso existe una clara intención de dañar al otro, ya sea física o moralmente, de tal suerte que la intimidación es constante y persiste a través del tiempo. Es muy notable el desequilibrio de fuerzas entre el agresor y la víctima, especialmente porque a ésta última se le dificulta sobreponerse a las burlas o agresiones y decide callar. En general la intimidación se define como un conjunto de acciones agresivas, intencionadas y repetitivas que causan dolor, angustia y sufrimiento (Smith, Cowie, Olafsson \& Liefooghe, 2002).

Las vías por las que se produce el bullying desde cuando se documentó, han evolucionado en el tiempo y han surgido nuevas formas de intimidación, acoso y malos tratos. Una de estas formas conocida como ciberbullying (Ortega, Calmaestra y Mora., 2008) ocurre al trascender los límites de la escuela para proyectarse a otros ámbitos a través de los medios virtuales, los cuales pueden contener grandes cantidades de información y llegar a millones de personas simultáneamente, situación difícil de detectar por parte de los profesores.

Online bullying o ciberbullying (también conocido como acoso cibernético) es una modalidad de agresión que comprende una serie de comportamientos repetidos con el fin de burlarse, menospreciar, o acosar a alguien más débil a través de la intimidación usando la internet, generalmente de forma anónima y potencialmente transmitido a una amplia audiencia. Se manifiesta principalmente en forma de mensajes de texto recibidos en el teléfono celular o móvil; fotografías o vídeos realizados con las cámaras de los móviles y posteriormente enviadas o usadas para amenazar a la víctima; llamadas al teléfono móvil acosadoras; e-mails insultantes o amenazantes; salas de chat en las que se agrede a uno de los participantes o se le excluye socialmente; acoso mediante los programas de mensajería instantánea (a través de programas como el Messenger); y páginas Web donde se difama a la víctima, se "cuelga" información personal o se hacen concursos en los que se ridiculiza a los demás (Ortega, et. al., 2008).

El bullying y el ciberbullying producen gran impacto en la sociedad en general, porque las acciones negativas, intencionadas e injustificadas de unas personas sobre otras, sitúan a las víctimas en una posición de la que difícilmente pueden escapar por sus propios medios y el no poder identificar a los agresores produce sentimientos de impotencia. En el caso de los niños, la continuidad de estas relaciones provoca efectos claramente negativos: ansiedad, descenso de la 
autoestima y cuadros depresivos, que dificultan su integración en el medio escolar y el desarrollo normal de los aprendizajes y en algunos casos la severidad del daño psicológico los conduce al suicidio (Ramírez, Álvarez, Valencia y Tirado, 2012; García, Velázquez, Martínez y Llanes, 2011).

Según Garaigordobil (2011), las ciber-víctimas tienen sentimientos de ansiedad, depresión, ideación suicida, estrés, miedo, baja autoestima, sentimientos de ira, frustración e indefensión, nerviosismo, irritabilidad, somatizaciones, trastornos del sueño y dificultades para concentrarse, que afectan al rendimiento escolar; los ciber-agresores por su parte muestran falta de empatía, conducta agresiva y delictiva, superior consumo de alcohol y drogas, dependencia de las tecnologías y absentismo escolar, entre otros.

Los/as espectadores/as, no permanecen indemnes. Al observar el comportamiento de sus compañeros se produce un aprendizaje vicario sobre cómo comportarse ante un conflicto, se refuerza el individualismo o el egoísmo y lo que es más peligroso, terminan valorando como importante y respetable la conducta agresiva. Se señala como consecuencia para ellos/as la desensibilización ante el sufrimiento de otros, a medida que van contemplando acciones repetidas de agresión en las que no son capaces de intervenir para evitarlas y en algunos casos podrían experimentar una sensación de indefensión semejante a la de la víctima (Barría, Matus, Cartagena, Mercado \& Mora, 2004).

Con base en las investigaciones existentes, Vandebosch, Beirens, D'Haese, Wegge \& Denis, (2012), sostienen que el ciberbullying es un fenómeno común entre los jóvenes y los padres, las escuelas, los proveedores de servicios de Internet o la policía son actores importantes en la prevención.

Desde la década del 2000 se han adelantado numerosos estudios dirigidos a establecer la presencia del Ciberbullying entre niños y adolescentes o externamente con personas especialmente adultas mediante la modalidad de grooming. Entre estos se encuentra por ejemplo el estudio realizado por Inglés, Hidalgo y Méndez (2000), quienes elaboraron y validaron el Cuestionario de Evaluación de Dificultades Interpersonales en la Adolescencia (CE- DIA), un autoinforme compuesto por 39 ítems distribuidos en cinco factores: aserción, relaciones con el otro sexo, relaciones con iguales, hablar en público y relaciones familiares. Buelga y Musitu, (2010) analizaron la prevalencia de la victimización a través del teléfono móvil y de Internet y establecieron las diferencias de género sobre el tipo de agresiones electrónicas sufridas, utilizando una escala de victimización entre iguales, ampliamente empleada y validada en España (Cava, Musitu \& Murgui, 2007). Se elaboraron las escalas para medir el acoso experimentado a través del teléfono móvil y de Internet y se seleccionaron y adaptaron a la situación de acoso tecnológico aquellos ítems que mejor representaban conceptualmente cada categoría de la clasificación de Willard (2006). Ortega et. al., (2008), exploraron el ciberbullying aplicando un cuestionario a un total de 830 escolares con edades comprendidas entre los 12 y 18 años. Los resultados mostraron que uno de cada cuatro escolares está involucrado en problemas de ciberbullying, aunque solamente un porcentaje, no superior al 4\%, estaría implicado 
de forma severa. El cuestionario utilizado "Cuestionario Ciberbullying" (Ortega, Calmaestra y Mora, 2007) está inspirado en el cuestionario diseñado por Smith, Mahdavi, Carvalho \& Tippett, (2006) pero agrupa las siete dimensiones originales en dos: episodios de ciberbullying utilizando el teléfono móvil y episodios de ciberbullying utilizando Internet (Ortega, et. al., 2008).

Con el fin de analizar la producción de instrumentos para evaluar ciberbullying, Berne, Frisén, Schultze-Krumbholz, Scheithauer, Naruskov, Luik, Katzer, Erentaite, Zukauskiene (2013) realizaron una revisión de publicaciones editadas entre 2004 y 2010 , que generó 636 citas, de las cuales seleccionaron un total de 41 instrumentos que cumplieron los criterios de haber sido publicados en idioma ingles y contener algunos de los siguientes términos: cyberbullying, cibervictimización, acoso cibernético o ciberagresión. Estos investigadores concluyeron que aunque se han desarrollado varios instrumentos para evaluar cyberbullying, faltan conocimientos sobre sus propiedades estructurales y psicométricas, tales como la validez y la fiabilidad, así como las definiciones y bases conceptuales. La información sobre la validez convergente se incluyó en sólo 24 de los 44 instrumentos, el concepto de acoso cibernético sólo se incluyó en 21 instrumentos y el de cibervictimización en 24 de los 44 instrumentos encontrados, de lo cual se infiere que el desarrollo de instrumentos de cyberbullying se ve obstaculizada por la aparente falta de consenso en cuanto a cuáles criterios se deben aplicar en la definición del término, lo que hace necesaria una operacionalización coherente del concepto "acoso cibernético", requisito necesario para que los investigadores puedan medir el mismo fenómeno a nivel nacional e interculturalmente (Berne, et. al. 2013).

\section{EVALUACIÓN DEL CIBERBULLYING}

Las mediciones psicológicas como las de cualquier otra ciencia, deben ser confiables y válidas; en términos generales un instrumento se considera confiable si las medidas que se hacen con este, carecen de errores de medida y son consistentes. La validez se refiere al conjunto de pruebas y datos que han de recogerse para garantizar la pertinencia de las inferencias que se hagan con los resultados; más que el test, lo que se validan son las inferencias. El problema de hallar la validez de un test es el problema general de la ciencia para validar una teoría, implica por tanto, la utilización de los métodos y procedimientos habituales de la investigación científica (Muñiz, 1998).

La International Test Commission (2010) exige tener en cuenta cuatro elementos básicos en la construcción de una prueba: el contexto (ámbito sociocultural de la aplicación), la construcción del test (creación y verificación de los ítems), la aplicación (control de fuentes de error), y la interpretación (documentación del análisis de datos y puntuaciones finales), aspectos tenidos en cuenta en el presente trabajo.

En el diseño y análisis del instrumento se utilizó tanto la TCT como la TRI, específicamente el modelo de Rasch. Bajo La TCT se estableció la consistencia 
interna, la validez de contenido y la validez de constructo; bajo el modelo de Rasch, se comprobó el ajuste de los ítems al modelo.

El instrumento elaborado, identifica los indicadores del constructo denominado "ciberbullying" descritos en el marco conceptual, limitado al ámbito escolar entre iguales. Por lo tanto quedan excluidas las agresiones, acoso o intimidación producidas por personas extrañas de cualquier edad o condición diferente a los compañeros de estudio.

Las características de las agresiones planteadas en cada uno de los ítems, se limitan al ámbito interpersonal, intrapersonal y contextual, omitiendo comportamientos intergrupales. Se tienen en cuenta las características definidas por Mendoza (2011) en cuanto que la conducta denote agresividad, sea negativa, se realice en múltiples ocasiones, exista desequilibrio de poder entre las partes, sea intencional, deliberada y cumpla un propósito. Tanto los agresores como las víctimas o los espectadores pueden ser detectados si señalan las opciones "Muy frecuentemente" o "Frecuentemente" en uno o más ítems. Las demás opciones solamente constituyen indicios de "ciberbullying" potencial o incipiente.

\section{Método}

\section{Tipo de investigación}

La presente investigación es instrumental de acuerdo con la categorización de Montero y León (2007), en cuanto estuvo encaminada al diseño y análisis de las propiedades psicométricas de una prueba, que permita establecer la presencia - ausencia de cyberbullying en un contexto escolar.

\section{Participantes}

Los participantes fueron 299 jóvenes estudiantes de sexo masculino y femenino, quienes se encontraban cursando los grados $6^{\circ}$ a $11^{\circ}$ en colegios públicos y privados de la ciudad de Bogotá, seleccionados aleatoriamente entre los escolares de las instituciones que decidieron participar.

\section{Instrumentos}

Se utilizó una Encuesta de Datos Sociodemográficos y el instrumento de Ciberbullying Escolar producto de la presente investigación. Con la encuesta socio-demográfica se lograron dos objetivos: caracterizar el grupo de participantes mediante el análisis de las principales variables sociodemográficas y asociar el ciberbullying con cada una de estas para obtener indicios acerca de los factores de riesgo. Las variables incluidas en la encuesta fueron: sexo, edad, estrato, estructura familiar, escolaridad de los padres, etnia, religión y acceso a la tecnología. El instrumento quedó conformado por 18 ítems que deben ser respondidos mediante escala Likert con 6 niveles de respuesta que van desde "Muy 
frecuentemente", "Frecuentemente", "Pocas veces", "Rara vez" hasta "nunca" y "No aplica".

\section{Descripción de los items}

Los primeros seis (6) ítems se refieren a conductas típicas de un agresor respecto a la utilización de las redes sociales para molestar, intimidar, amenazar, ridiculizar e incomodar a los demás mediante comunicaciones hostiles o la publicación de materiales ofensivos. Siete (7) ítems están relacionados con percepciones de quienes han sido agredidos a través de redes sociales, los cuales permiten identificar a las víctimas. Los cinco (5) ítems restantes corresponden a conductas típicas de los espectadores al actuar en forma pasiva o involucrándose de algún modo. Si el examinado responde uno o más de los ítems en el nivel "Frecuentemente" o "Muy frecuentemente" se considera que pertenece al respectivo rol.

\section{Procedimiento}

En la primera fase se determinaron las características básicas del cyberbullying, a partir de las cuales se desarrolló el plan de prueba. La segunda fase consistió en el diseño de la prueba, para lo cual se elaboró un primer instrumento. En la tercera fase el instrumento o prueba se sometió a evaluación por parte de siete jueces expertos; a partir de dicha revisión, se identificaron algunos errores e inconsistencias y se realizaron los ajustes pertinentes para realizar el pilotaje. El instrumento corregido, fue aplicado a una muestra de 299 estudiantes. En la quinta fase se realizaron los análisis estadísticos y psicométricos en cinco aspectos: análisis descriptivos, análisis de consistencia interna, validez de contenido, validez de constructo y ajuste de los ítems al modelo.

\section{RESULTADOS}

En la distribución de frecuencias de los resultados de la prueba (Tabla 1), se determinó que el rol de agresores puede ser único o puede combinarse con otros roles como espectador o como víctima simultáneamente. Por lo tanto al sumar Agresor solo 0.33\%; Agresor - Espectador 6.02\% y Espectador - Agresor - Víctima 2.68, el total representa un 9.03\%. Del mismo modo, las víctimas cumplen simultáneamente otros roles, al sumar Víctima Solo 0.67\%; Víctima Espectador 9.36\% y Espectador - Agresor - Víctima 2.68\% el total representa un $12.71 \%$. Quienes han asumido el rol de espectadores corresponden al $37.12 \%$, mientras que quienes se hallan ajenos a este tipo de comportamiento corresponden al 43,81\%; de lo anterior se concluye que el $19.07 \%$ cumple un papel activo como agresor o víctima (resultado obtenido de restar al 100\% los porcentajes de las categorías ninguno y espectador sólo), pero como los espectadores también están involucrados de una u otra manera, el total de quienes realmente participan en ciberbullying es de $56,19 \%$ 
Tabla 1. Resultados de la Aplicación de la Prueba a la Muestra de Adolescentes

\begin{tabular}{lcc}
\hline Categoría & Frecuencia & Porcentaje \\
\hline Agresor solo & 1 & 0,33 \\
Víctima solo & 2 & 0,67 \\
Espectador solo & 111 & 37,12 \\
Agresor - Espectador & 18 & 6,02 \\
Víctima - Espectador & 28 & 9,36 \\
Espectador - Agresor - Víctima & 8 & 2,68 \\
Ninguno & 131 & 43,81 \\
\hline
\end{tabular}

\section{Análisis Psicométrico}

Para establecer la consistencia interna de la prueba se utilizó el alfa de Cronbach cuyo valor fue de 0.864 , con los 18 ítems que finalmente quedaron en la prueba final.

\section{Validez de contenido según la evaluación realizada por los expertos}

Se construyó el ICIB con 25 ítems iniciales según el plan de prueba, una vez revisado se sometió a prueba por 6 jueces; para obtener la validez de contenido se utilizó el índice $r_{w g}$ para evaluar pertinencia, coherencia, relevancia, sintaxis y semántica. Se encontró que se debían revisar los ítems 1, 11, 12, 13, 14, 15, 19 y 20 en cuanto a su pertinencia, en todos los demás aspectos los ítems fueron bien evaluados. Se llevó a cabo la revisión de cada uno de los ítems, siguiendo las indicaciones sugeridas por los expertos y las puntuaciones del índice $r_{w g}$, se eliminaron los ítems $4,8,9,10,16,18$ y 23 puesto que en todos estos ítems, el índice $r_{w g}$ fue inferior a 0.50 , lo cual indicaba desacuerdo entre los jueces. El ICIB final quedó compuesto por 18 ítems.

\section{Validez de constructo}

Antes de llevar a cabo el análisis factorial exploratorio, se aplicó la prueba Kaiser-Meyer-Olkin (KMO) con el objetivo de estimar si el tamaño de la muestra era adecuado para realizar el análisis, también se estimó la homogeneidad de la varianza de la muestra con la prueba de esfericidad de Bartlett. La prueba KMO obtuvo un valor de 0.846 que indica que el tamaño de la muestra es adecuado para realizar el análisis y la prueba de Bartlett dio significativa $(p=0.00)$, lo que permitió realizar el análisis.

Se llevó a cabo el análisis factorial exploratorio en el SPSS versión 21, con el método de componentes principales y rotación varimax, el cual arrojó tres factores que explican el $49,43 \%$ de la varianza total. Los resultados se muestran 
en la Tabla 2.

Tabla 2. Distribución Factorial de los Ítems

\begin{tabular}{|c|c|c|c|}
\hline \multirow[b]{2}{*}{ ITEMS } & \multicolumn{3}{|c|}{ FACTORES } \\
\hline & 1 & 2 & 3 \\
\hline 1. Utilizo redes sociales para intimidar a otros amenazándolos. & & ,526 & \\
\hline 2. Publico en las redes sociales los defectos de quienes me caen mal & & 485 & \\
\hline $\begin{array}{l}\text { 3. He subido imágenes ridículas en las redes sociales para burlarme } \\
\text { de otros. }\end{array}$ & & 618 & \\
\hline 5. Encuentro la manera de molestar a otros en las redes sociales & & ,712 & \\
\hline 6. Me divierto ridiculizando a otros a través de las redes sociales & &, 836 & \\
\hline $\begin{array}{l}\text { 7. Tengo la satisfacción de haberme burlado de otros a través de } \\
\text { redes sociales }\end{array}$ & & ,659 & \\
\hline $\begin{array}{l}\text { 11. He sido perjudicado con información falsa que han publicado } \\
\text { sobre mí en las redes sociales. }\end{array}$ & ,639 & & \\
\hline $\begin{array}{l}\text { 12. Alguien hackeó mi Facebook y escribe cosas a nombre mío } \\
\text { para hacerme quedar mal ante los demás. }\end{array}$ & ,603 & & \\
\hline $\begin{array}{l}\text { 13. Alguien ha publicado en las redes sociales información acerca } \\
\text { de mi vida privada }\end{array}$ &, 581 & & \\
\hline 14. He recibido mensajes de texto ofensivos en mi celular & ,635 & & \\
\hline 15. He sido amenazado (a) en las redes sociales & 665 & & \\
\hline $\begin{array}{l}\text { 17. Me siento muy mal por las agresiones que he recibido por las } \\
\text { redes sociales }\end{array}$ & ,683 & & \\
\hline $\begin{array}{l}\text { 19. Mis compañeros saben que en las redes sociales algunas } \\
\text { personas se burlan de mi }\end{array}$ & ,662 & & \\
\hline 20. Algunos compañeros usan Facebook para agredir a otros & & & ,756 \\
\hline $\begin{array}{l}\text { 21. Apoyo a mis compañeros cuando sé que los están molestando } \\
\text { por internet }\end{array}$ & & & ,465 \\
\hline 22. Observo con interés las peleas que ocurren en las redes sociales & & & ,548 \\
\hline $\begin{array}{l}\text { 24. Mis compañeros se involucran en las peleas de los demás en las } \\
\text { redes sociales aunque no tengan nada que ver con ellos }\end{array}$ & & & ,752 \\
\hline $\begin{array}{l}\text { 25. Existe indiferencia entre mis compañeros cuando agreden a } \\
\text { otros en las redes sociales }\end{array}$ & & & ,792 \\
\hline
\end{tabular}

Los ítems 1, 2, 3, 5, 6 y 7, evalúan los aspectos que se refieren al agresor, los ítems $11,12,13,14,15,17$ y 19 se refieren a la víctima y los ítems 20,21, 22, 24 y 25 a los espectadores.La tabla 3 presenta los estadísticos descriptivos de cada uno de los ítems, analizando la asimetría y la curtosis se deduce que sólo el ítem 1 , no se distribuye de forma normal, esto se debe a que el $90 \%$ de los participantes marcaron la opción 1. La moda de todos los ítems fue 1, excepto la del ítem 20 , cuyo puntaje fue 2 , las desviaciones estándar oscilaron entre 0,34 y 0,78 . 
Tabla 3. Descriptivos de Cada Uno de los Items

\begin{tabular}{lcccccc}
\hline \multicolumn{1}{c}{ Ítem } & Media & Mediana & Moda & Desv. típ. & Asimetría & Curtosis \\
\hline P1 & 1,11 & 1,00 & 1,0 &, 34 & 3,21 & 10,40 \\
P2 & 1,23 & 1,00 & 1,0 &, 44 & 1,55 & 1,08 \\
P3 & 1,34 & 1,00 & 1,0 &, 52 & 1,18 &, 35 \\
P5 & 1,37 & 1,00 & 1,0 &, 57 & 1,23 &, 52 \\
P6 & 1,26 & 1,00 & 1,0 &, 47 & 1,58 & 1,52 \\
P7 & 1,27 & 1,00 & 1,0 &, 49 & 1,57 & 1,53 \\
P11 & 1,37 & 1,00 & 1,0 &, 61 & 1,39 &, 85 \\
P12 & 1,20 & 1,00 & 1,0 &, 47 & 2,37 & 4,97 \\
P13 & 1,13 & 1,00 & 1,0 &, 35 & 2,70 & 6,74 \\
P14 & 1,17 & 1,00 & 1,0 &, 42 & 2,38 & 5,14 \\
P15 & 1,27 & 1,00 & 1,0 &, 50 & 1,61 & 1,68 \\
P17 & 1,23 & 1,00 & 1,0 &, 50 & 2,09 & 3,58 \\
P19 & 1,23 & 1,00 & 1,0 &, 50 & 2,05 & 3,43 \\
P20 & 2,08 & 2,00 & 2,0 &, 77 &,- 14 & $-1,31$ \\
P21 & 1,71 & 2,00 & 1,0 &, 76 &, 55 & $-1,09$ \\
P22 & 1,84 & 2,00 & 1,0 &, 78 &, 29 & $-1,31$ \\
P24 & 1,79 & 2,00 & 1,0 &, 77 &, 37 & $-1,21$ \\
P25 & 1,79 & 2,00 & 1,0 &, 75 &, 37 & $-1,16$ \\
\hline
\end{tabular}

Bajo la TRI, los indicadores de medición OUTFIT e INFIT de cada ítem estuvieron dentro de los parámetros recomendados por diversos autores (v.g. Muñiz, 2010; Attorresi, Lozzia, Abal, Galibert, \& Aguerri, 2009; Barbosa \& Reyes, 2007; Kohan, 2004). Al evaluar los ítems mediante la TRI se obtuvo un alto ajuste al modelo de Rash mediante los indicadores INFIT y OUTFIT en los tres factores donde el estadígrafo toma valores de rango -2 a +2 , los cuales están en el intervalo de lógitos aceptable para determinar ajuste razonable. Únicamente un valor superior a +2 en ZSTD $(2,6)$ se halla en el ítem 21 "Apoyo a mis compañeros cuando sé que los están molestando por internet" lo cual puede ser un indicador de falta de ajuste del ítem, que puede deberse al diseño del mismo o a la forma en que los estudiantes lo interpretaron. (Tabla 4) 
Tabla 4. Estadígrafos de Ajuste del Modelo Rasch para los Reactivos del ICIB

\begin{tabular}{lllll}
\hline Ítem & $\mathbf{N}$ & Error & INFIT & OUTFIT \\
\hline Item1 & 299 & 0.10 & 1.06 & 1.04 \\
Item2 & 299 & 0.10 & 1.19 & 1.24 \\
Item3 & 299 & 0.09 & 1.03 & 0.94 \\
Item5 & 299 & 0.08 & 0.92 & 0.97 \\
Item6 & 299 & 0.09 & 0.73 & 0.74 \\
Item7 & 299 & 0.09 & 1.03 & 0.99 \\
Item11 & 299 & 0.07 & 1.0 & 1.17 \\
Item12 & 299 & 0.09 & 1.13 & 1.21 \\
Item13 & 299 & 0.10 & 1.0 & 1.01 \\
Item14 & 299 & 0.09 & 0.96 & 0.86 \\
Item15 & 299 & 0.09 & 0.92 & 0.88 \\
Item17 & 299 & 0.08 & 0.86 & 0.90 \\
Item20 & 299 & 0.05 & 0.96 & 0.97 \\
Item21 & 299 & 0.06 & 1.23 & 1.18 \\
Item22 & 299 & 0.05 & 1.03 & 1.02 \\
Item24 & 299 & 0.06 & 0.91 & 0.91 \\
Item25 & 299 & 0.06 & 0.91 & 0.88 \\
\hline
\end{tabular}

Nota: N (participantes), INFIT(Estadístico estandarizado de ajuste cercano), OUTFIT(Estadístico estandarizado de ajuste lejano), Fuente: elaboración propia.

El siguiente paso consistió en relacionar los resultados del ICIB con las variables psicosociales y de uso de la tecnología, cuyos resultados revelaron que existe asociación significante entre las variables "destreza en el manejo de herramientas informáticas" como Diseño de Blogs, Photoshop, Edición de Vídeo, Paint y Música con los estudiantes involucrados en cyberbullying; estas herramientas se consideran más avanzadas que el Office. La tabla 5 presenta los porcentajes promedio de los involucrados y no involucrados en Ciberbullying en cada una de las herramientas virtuales, la mayor diferencia se encuentra en edición de música, en promedio $76,9 \%$ para los involucrados, frente a $23,1 \%$ para los no involucrados. 
Tabla 5. Asociación entre Variables. Manejo de Informática y Ciberbullying

\begin{tabular}{ccccc}
\hline $\begin{array}{c}\text { Herramienta } \\
\text { virtual }\end{array}$ & $\begin{array}{c}\text { \% Promedio } \\
\text { involucrados } \\
\text { en } \\
\text { ciberbullying }\end{array}$ & $\begin{array}{c}\text { \% Promedio no } \\
\text { involucrados } \\
\text { en } \\
\text { ciberbullying }\end{array}$ & Chi cuadrado & $\mathrm{p}$ \\
\hline Vídeo & 60,72 & 37,45 & 5,82 & 0,01 \\
Blogs & 73,22 & 34,4 & 10,81 & 0,01 \\
Photoshop & 82,26 & 45,75 & 13,27 & 0,00 \\
Paint & 54,7 & 45,3 & 14,42 & 0,00 \\
Música & 76,9 & 23,1 & 4,97 & 0,03 \\
\hline
\end{tabular}

La asociación entre las variables "número de equipos que posee para su uso personal" (Dos o menos equipos y tres o más equipos) y categorías del cyberbullying, cuyas categorías incluyen no está involucrado (espectador o ninguno) y si está involucrado (todas las demás categorías); resultó estadísticamente significante, el valor del estadístico exacto de Fisher fue 4.65 con un valor sig bilateral de 0.03 (Tabla 6).

Tabla 6. Asociación entre Variables. Número de Equipos que Posee para Su Uso Personal y Categorías del Cyberbullying

\begin{tabular}{cccc}
\hline $\begin{array}{c}\text { Número de equipos } \\
\text { que posee para su } \\
\text { uso personal }\end{array}$ & \multicolumn{3}{c}{ Ciberbullying } \\
\hline \multirow{2}{*}{$\begin{array}{c}\text { Dos o menos } \\
\text { equipos }\end{array}$} & No está involucrado & Si está involucrado & Total \\
\cline { 2 - 4 } & 136 & 23 & 159 \\
\cline { 2 - 4 } & $56,2 \%$ & $40,4 \%$ & $53,2 \%$ \\
Tres o más equipos & 106 & 34 & 140 \\
& $43,8 \%$ & $59,6 \%$ & $46,8 \%$ \\
\cline { 2 - 4 } Total & 242 & 57 & 299 \\
& $100,0 \%$ & $100,0 \%$ & $100,0 \%$ \\
\hline
\end{tabular}


De la tabla 6 se deduce que del total de estudiantes que están involucrados con el ciberbullying, el 59.6\% tiene para su uso personal más de tres equipos entre teléfono móvil, Smartphone, tableta, computadora portátil o computadora fija; este porcentaje entre los jóvenes que tienen dos o menos equipos, corresponde al $40.4 \%$.

\section{DISCUSIÓN}

Según los resultados obtenidos se puede afirmar que se lograron los objetivos propuestos ya que el análisis psicométrico del "ICIB" indicó estructura factorial adecuada y el análisis factorial exploratorio arrojó tres factores que identificaron claramente los conjuntos de ítems relativos a agresor, víctima y espectador, que era lo esperado.

La intimidación en línea afecta a uno de cada cuatro niños de 12-18 años de edad (Ortega et. al. 2008), indicador que se aproxima a los resultados obtenidos en el presente estudio en el cual se encontró que el 21,74\% de los jóvenes se ha visto involucrado en ciberbullying bien sea como agresor o como víctima. Sin embargo es importante señalar que la incidencia puede ser mayor, si se considera a los espectadores como partícipes activos del hostigamiento en las escuelas. Tales resultados deben ser contrastados en el futuro con mediciones para el resto de la población colombiana mediante la elección de muestras representativas.

En el presente estudio, el total de agresores representó un 9,03\%, indicador que debe ser tenido en cuenta; de acuerdo con Barría, Matus, Cartagena, Mercado y Mora (2004), tal comportamiento es un preámbulo de una serie de problemas en su vida futura porque los agresores están sujetos a consecuencias indeseadas y puede suponer para él/ella un aprendizaje sobre cómo conseguir los objetivos y, por tanto, estar en antesala de la conducta delictiva.

Las principales fortalezas del instrumento radican en una adecuada formulación de las bases conceptuales, resultados coherentes con lo esperado y análisis de sus propiedades estructurales y psicométricas, tales como la fiabilidad, validez de contenido, convergente y de constructo, aspectos que no fueron encontrados en la revisión del alto volumen de estudios revisados por Berne et al., (2013) que no incluyen estos criterios. Con el presente estudio se aporta en el cumplimiento de requisitos esenciales del diseño de instrumentos para evaluar ciberbullying, aunque queda pendiente avanzar en la operacionalización del constructo de una manera más precisa.

Un hallazgo importante para analizar en futuras investigaciones, es la relación entre destrezas informáticas o la disponibilidad de instrumentos tecnológicos y la participación activa en ciberbullying. 
Se concluye que El ICIB, instrumento conformado finalmente por 18 ítems, permitirá detectar la presencia del fenómeno en contextos escolares y establecer el rol de cada participante, lo cual facilitará la implementación de programas educativos acordes con cada situación. Se sugiere analizar las propiedades psicométricas a la luz de estudios confirmatorios posteriores, que garanticen una medida mucho más precisa del constructo.

\section{AGRADECIMIENTOS}

Especial agradecimiento a la Universidad Católica de Colombia por haber apoyado la presente investigación

\section{REFERENCIAS}

Attorresi, H. F., Lozzia, G. S., Abal, F. J., Galibert, M. S., \& Aguerri, M. E. (2009). Teoría de Respuesta al Ítem. Conceptos básicos y aplicaciones para la medición de constructos psicológicos. Revista Argentina de Clínica Psicológica, 18(2), 179-188.

Barbosa, Y., \& Reyes, J. F. (2007). MINISTEPS. Obtenido de Universidad Nacional de Colombia. Facultad de Ciencias Económicas. Unidad de informática y comunicaciones: Recuperado de: http://fce.unal.edu.co/wiki/images/2/2d/ MiniSteps.pdf

Barría, P., Matus, C., Cartagena, D., Mercado, Y., \& Mora C. (2004) Bullying y Rendimiento Escolar, Tesis Universidad Católica de Temuco Facultad de Educación.

Berne, S., Frisén, A., Schultze-Krumbholz, A., Scheithauer, H., Naruskov, K., Luik, P., Katzer, C., Erentaite, R., Zukauskiene R., 3013, Cyberbullying assessment instruments: A systematic review. Aggression and Violent Behavior $18,320-334$

Buelga, S., \& Musitu, G. (2010). Ciberbulling: victimización entre adolescentes a través del teléfono móvil y de Internet. Psicothema, 22 (4), 784-789.

Cava, M., Musitu, G., \& Murgui, S. (2007). Individual and social risk factors related to overt victimization in a sample of Spanish adolescents. Psychological Reports, 101, 275-290.

Garaigordobil, M. (2011) Prevalencia y consecuencias del cyberbullying: una revisión International Journal of Psychology and Psychological Therapy 11 (2), 233-254.

García, G., Velázquez, J., Martínez, G. y Llanes, A. (2011). Ciberbullying: forma virtual de intimidación escolar. Revista Colombiana de Psiquiatría, 3, 115130.

Inglés, C., Hidalgo, M. \& Méndez, J. (2000). Cuestionario de Evaluación de Dificultades Interpersonales en la Adolescencia Psicothema, 12 (3), 390-398

International Test Commission. (2010). The ITC Guidelines for translating and adapting test. Recuperado de http://www.intestcom.org/upload/sitefiles/40. 
pdf

Kohan, N. (2004). Teoría de Respuesta al Ítem: Supuestos Básicos. Evaluar, 4, 95-110.

Mendoza, M. T (2011) La violencia en la escuela. Bullies y víctimas. México, Trillas.

Montero, I., y León, O. (2007). Guía para nombrar los estudios de investigación en Psicología. International Journal of Clinical and Health Psychology, 7, 847-862.

Muñiz, J. (1998). Teoría Clásica de los Tests. Madrid: Pirámide.

Muñiz, J. (2010). Las teorías de los test: Teoría clásica y teoría de respuesta a los ítems. Papeles del Psicólogo, 31(1), 57-66.

Olweus, Dan. (1998). Conductas de Acoso y Amenaza entre Escolares. Ediciones Morata. Madrid. 1998.

Ortega, R., Calmaestra, J. \& Mora, J. (2008). Ciberbulling International. Journal of Psychology and Psychological Therapy, 8 (2) 183-192

Ortega, R., Calmaestra, J. y Mora, J. (2007). Cuestionario Cyberbullying. Universidad de Córdoba, instrumento no publicado.

Ramírez, R., Álvarez, C., Valencia G. yTirado, F. (2012). Prevalencia de sintomatología depresiva y ansiosa en estudiantes de 14 a 18 años de un colegio privado de Medellín. Revista Colombiana de Psiquiatría, 41 (3), 606-619

San Martín, J., (2007). Violencia y Acoso Escolar. Revista Mente y Cerebro, 26, 12-19.

Smith, P., Mahdavi J., Carvalho, C. \& Tippett, N. (2006). An investigation into ciberbulling, its forms, awareness and impact, and the relationship between age and gender in ciberbulling. A Report to the Anti-Bullying Alliance.

Smith, P., Cowie, H., Olafsson, R., \& Liefooghe, A. (2002). Definitions of bullying: A comparison of terms used, and age and gender differences, in a fourteen-country international comparison. Child Development, 73(4), 11191133 .

Vandebosch, H., Beirens, L., D’Haese, W., Wegge \& Denis, S. (2012) Police actions with regard to ciberbulling: The Belgian case. Psicothema, 24 (4) 646-652

Willard, N. (2006). Cyberbullying and Cyberthreats. Effectively Managing Internet Use Risks in Schools. Recuperado de: http://new.csriu.org/cyberbully/ docs/cbctpresentation.pdf 